\title{
Effect of Herbicides on Soil Microflora and Dehydrogenase Activity in Transplanted Bt Cotton Based Intercropping System
}

\author{
K. R. Siddagangamma*, A. S. Channabasavanna, Mahadevaswamy, \\ K. Narayana Rao, M. Y. Ajayakumar and G. S. Yadahalli \\ College of Agriculture, University of Agricultural Sciences, \\ Raichur-584104, Karnataka, India \\ *Corresponding author
}

Keywords

Pendimethalin, Oxadiargyl, Bacteria, Fungi, Actinomycetes and Dehydrogenase enzyme activity

Article Info

Accepted: 12 December 2020 Available Online: 10 January 2021
The experiment was conducted to analyse the effect of herbicides on soil microflora and Dehydrogenase activity in transplanted $B t$ cotton based intercropping system during 201819 and 2019-20, at Agricultural Research Station, Malnoor, University of Agricultural Sciences, Raichur, Karnataka. The soil of the experimental field was medium deep black with clay loam texture, slightly high in $\mathrm{pH}(8.14)$, normal EC $\left(0.35 \mathrm{dS} \mathrm{m}^{-1}\right)$, medium in OC $(0.56 \%)$, available nitrogen $\left(344 \mathrm{~kg} \mathrm{ha}^{-1}\right)$, phosphorus $\left(29.21 \mathrm{~kg} \mathrm{ha}^{-1}\right)$ and high in available potassium $\left(355 \mathrm{~kg} \mathrm{ha}^{-1}\right)$. The treatments consisted of four cropping systems in main plots and five weed control treatments in sub plots and were replicated thrice in a split plot design. The data indicated that pre emergence application of pendimethalin 38.7 CS @ $0.34 \mathrm{~kg}$ a.i. ha ${ }^{-1}$ or oxadiargyl @ $0.04 \mathrm{~kg}$ a.i. ha ${ }^{-1}$ reduced the microbial population (Bacteria, Fungi and Actinomycetes) and Dehydrogenase enzyme activity significantly over the treatments where no herbicides were applied (unweeded check, weed free, hand weeding). While the values were on par at 60 DAS and at harvest which indicated that the herbicide effect did not persist for longer time as the herbicides degraded in soil by microbes as the herbicides were used as the carbon source for multiplication. The seed cotton equivalent yield was higher when herbicides were used, indicating that no adverse effect of the herbicide.

\section{Introduction}

Cotton the "white gold or the king of fibres" is one of the most important commercial crop. Cotton is known for the fibre and oil from seed, which plays a prominent role in the national and international economy. The Initial slow growth and adoption of wider spacing offers ample scope for raising intercrops viz., foxtail millet and little millet.
Intercropping in transplanted $B t$ cotton an economical approach for higher unit productivity from same piece of land. In cropping systems, weeds often pose a major threat to crop yields, manual weeding is always not feasible because of increasing labour cost and also due to scarcity of human labour. Hence it is necessary to use herbicides to control weeds in intercropping system. Though it is well know that use of herbicides 
controls weeds efficiently and it is more economical but on the other hand, they may have adverse effect on soil health and soil microflora. Hence, the present study was to investigate how agronomic practices and herbicide application in transplanted $B t$ cotton intercropped with foxtail millet and little millet would affect soil microbial population and Dehydrogenase activity.

\section{Materials and Methods}

The application of herbicides and its impact in intercropping systems on major soil microbial population and Dehydrogenase activity was studied at Agricultural Research Station, Malnoor, University of Agricultural Sciences, Raichur, during 2018-19 and 2019-20. The soils of the experimental field was medium deep black with clay loam texture, slightly high in $\mathrm{pH}(8.14)$, normal EC $\left(0.35 \mathrm{dS} \mathrm{m}^{-1}\right)$, medium in OC $(0.56 \%)$, available nitrogen (344 kg ha ${ }^{-1}$ ), phosphorus (29.21 $\mathrm{kg} \mathrm{ha}^{-1}$ ) and high in available potassium (355 kg ha $\left.{ }^{-1}\right)$. The treatments consisted of four cropping systems \{ sole $B t$ cotton $(90 \mathrm{~cm} \times 60 \mathrm{~cm}$ ), paired row planting of $B t$ cotton $(120 / 60 \mathrm{~cm} \mathrm{x} 60 \mathrm{~cm})$, paired row $B t$ cotton + foxtail millet $(2: 3)$ and paired row $B t$ cotton + little millet $(2: 3)\}$ in the main plots and five weed control treatments \{ unweeded check, weed free, hand weeding @ 20 DAS $f b$ IC at 35 DAT, pendimethalin 38.7 CS@0.34 kg a.i. ha ${ }^{-1}$ as PE $f b$ IC at 35 DAT and oxadiargyl @ 0.04 $\mathrm{kg}$ a.i. ha ${ }^{-1}$ as PE $f b$ IC at 35 DAT $\}$ in sub plots replicated thrice in a split plot design. For the purpose of analyzing the microbial activity, the soil samples were collected from experimental plot at 30, 60 DAT and at harvest of crop from each plot. The enumeration of total Bacteria, Fungi and Actinomycetes was carried out at different interval by serial dilution and Agar plate count method (Pramer and Schmidt, 1964). Dehydrogenase activity in the soil sample by colorimetric determination of TPF produced from the TTC in soils by Assay method as described by Casida et al., (1964) at a wave length of $485 \mathrm{~nm}$. The results are expressed as $\mu \mathrm{g}$ of triphenyl formazan (TPF) formed per gram of soil per day. The data were analyzed statistically as per the procedure described by Gomez and Gomez (1984).

\section{Results and Discussion}

\section{Soil Microbial population (Bacteria, Fungi and Actinomycetes)}

In both the seasons the data indicated that cropping systems did not affect the microbial population, where as the weed management practices influenced the microbial population significantly. In general, load of microbial population reduced immediately after the application of herbicides and as the days to go it attained normal state. Among weed management practices, the pooled data at 30 DAT indicated that application of pendimethalin $38.7 \mathrm{CS} @ 0.34 \mathrm{~kg}$ a.i. ha ${ }^{-1}$ as pre emergence recorded bacteria, fungi and actinomycetes population of $34.68 \times 10^{6} \mathrm{CFU}$ $\mathrm{g}^{-1}$ of soil, $22.02 \times 10^{3} \mathrm{CFU} \mathrm{g} \mathrm{g}^{-1}$ of soil and $17.12 \times 10^{4} \mathrm{CFU} \mathrm{g}^{-1}$ of soil, respectively and when oxadiargyl @ $0.04 \mathrm{~kg}$ a.i. ha ${ }^{-1}$ was applied as pre emergence it was $33.70 \times 10^{6}$ CFU g ${ }^{-1}$ of soil, $16.35 \times 10^{3} \mathrm{CFU} \mathrm{g}^{-1}$ of soil and $14.64 \times 10^{4} \mathrm{CFU} \mathrm{g}{ }^{-1}$ of soil, respectively. All these values were significantly lesser over plots where no herbicide was applied viz., unweeded check $\left(76.48 \times 10^{6} \mathrm{CFU} \mathrm{g}^{-1}\right.$ of soil, $32.16 \times 10^{3} \mathrm{CFU} \mathrm{g}^{-1}$ of soil and $45.92 \times 10^{4}$ CFU $\mathrm{g}^{-1}$ of soil, respectively), weed free check $\left(74.79 \times 10^{6} \mathrm{CFU} \mathrm{g}^{-1}\right.$ of soil, $31.47 \times$ $10^{3} \mathrm{CFU} \mathrm{g}{ }^{-1}$ of soil and $45.15 \times 10^{4} \mathrm{CFU} \mathrm{g}^{-1}$ of soil, respectively) and hand weeding @ 20 DAT $f b$ IC at 35 DAT $\left(67.61 \times 10^{6} \mathrm{CFU} \mathrm{g}^{-1}\right.$ of soil, $30.34 \times 10^{3} \mathrm{CFU} \mathrm{g}{ }^{-1}$ of soil and $42.81 \times$ $10^{4} \mathrm{CFU} \mathrm{\textrm {g } ^ { - 1 }}$ of soil, respectively). The latter treatments were on par with each other (Table 1-3). However, the data was non significant at 60 days after transplanting and at harvest. 
Table.1 Bacterial population (No. $\times 10^{6} \mathrm{CFU} \mathrm{g}^{-1}$ of soil) at different growth stages as influenced by weed management practices in transplanted $B t$ cotton with minor millet intercropping system

\begin{tabular}{|c|c|c|c|c|c|c|c|c|c|}
\hline \multirow{3}{*}{$\begin{array}{l}\text { Treatments } \\
\text { Cropping system }\end{array}$} & \multicolumn{9}{|c|}{ Bacterial population (No. $\times 10^{6} \mathrm{CFU} \mathrm{g}^{-1}$ of soil) } \\
\hline & \multicolumn{3}{|c|}{ 30 DAT } & \multicolumn{3}{|c|}{60 DAT } & \multicolumn{3}{|c|}{ At Harvest } \\
\hline & 2018 & 2019 & Pooled & 2018 & 2019 & Pooled & 2018 & 2019 & Pooled \\
\hline Sole $B t$ cotton $(90 \mathrm{~cm} \times 60 \mathrm{~cm})$ & 56.70 & 54.14 & 55.42 & 77.40 & 76.13 & 76.77 & 54.47 & 54.09 & 54.28 \\
\hline Paired row planting of $B t$ cotton $(120 / 60 \mathrm{~cm} \times 60 \mathrm{~cm})$ & 57.80 & 54.58 & 56.19 & 76.13 & 74.67 & 75.40 & 54.00 & 53.83 & 53.92 \\
\hline Paired row $B t$ cotton + Foxtail millet $(2: 3)$ & 63.04 & 56.90 & 59.97 & 81.13 & 77.53 & 79.33 & 58.70 & 56.24 & 57.47 \\
\hline Paired row $B t$ cotton + Little millet $(2: 3)$ & 61.35 & 55.07 & 58.21 & 79.07 & 76.60 & 77.83 & 55.23 & 55.10 & 55.16 \\
\hline S. Em \pm & 2.44 & 2.71 & 2.50 & 3.04 & 4.24 & 3.23 & 4.24 & 2.34 & 3.08 \\
\hline C.D. at $5 \%$ & NS & NS & NS & NS & NS & NS & NS & NS & NS \\
\hline \multicolumn{10}{|l|}{ Weed management practices } \\
\hline Un weeded check & 79.58 & 73.37 & 76.48 & 85.62 & 80.58 & 83.10 & 58.92 & 57.82 & 58.37 \\
\hline Weed free & 78.81 & 70.76 & 74.79 & 83.75 & 79.92 & 81.83 & 57.00 & 56.99 & 57.00 \\
\hline Hand weeding @ 20 DAS $f b$ IC at 35 DAT & 71.38 & 63.83 & 67.61 & 77.70 & 74.08 & 75.89 & 55.24 & 56.28 & 55.76 \\
\hline Pendimethalin 38.7 CS @ $0.34 \mathrm{~kg} a . i . \mathrm{ha}^{-1}$ as PE $f b$ IC at 35 DAT & 35.01 & 34.34 & 34.68 & 73.77 & 73.42 & 73.59 & 54.92 & 52.41 & 53.66 \\
\hline Oxadiargyl @ $0.04 \mathrm{~kg} a . i \mathrm{ha}^{-1}$ as PE $f b$ IC at 35 DAT & 33.83 & 33.56 & 33.70 & 71.33 & 73.17 & 72.25 & 51.92 & 50.58 & 51.25 \\
\hline S. Em \pm & 2.67 & 2.83 & 2.59 & 3.96 & 3.07 & 2.99 & 3.59 & 3.26 & 3.06 \\
\hline C.D. at $5 \%$ & 7.69 & 8.15 & 7.47 & NS & NS & NS & NS & NS & NS \\
\hline
\end{tabular}

Table.2 Fungal population (No. $\times 10^{3} \mathrm{CFU} \mathrm{g}^{-1}$ of soil) at different growth stages as influenced by weed management practices in transplanted $B t$ cotton with minor millet intercropping system

\begin{tabular}{|c|c|c|c|c|c|c|c|c|c|}
\hline \multirow{3}{*}{$\begin{array}{l}\text { Treatments } \\
\text { Cropping system }\end{array}$} & \multicolumn{9}{|c|}{ Fungal population (No. $\times 10^{3} \mathrm{CFU} \mathrm{g}^{-1}$ of soil) } \\
\hline & \multicolumn{3}{|c|}{30 DAT } & \multicolumn{3}{|c|}{60 DAT } & \multicolumn{3}{|c|}{ At Harvest } \\
\hline & 2018 & 2019 & Pooled & 2018 & 2019 & Pooled & 2018 & 2019 & Pooled \\
\hline Sole $B t$ cotton $(90 \mathrm{~cm} \times 60 \mathrm{~cm})$ & 25.99 & 26.09 & 26.04 & 33.08 & 31.62 & 32.35 & 12.35 & 11.40 & 11.87 \\
\hline Paired row planting of $B t$ cotton $(120 / 60 \mathrm{~cm} \times 60 \mathrm{~cm})$ & 26.96 & 22.87 & 24.91 & 33.62 & 31.39 & 32.50 & 11.64 & 10.66 & 11.15 \\
\hline Paired row $B t$ cotton + Foxtail millet $(2: 3)$ & 30.20 & 30.13 & 30.17 & 34.70 & 35.36 & 35.03 & 12.84 & 12.29 & 12.57 \\
\hline Paired row $B t$ cotton + Little millet $(2: 3)$ & 25.84 & 23.67 & 24.75 & 33.67 & 32.31 & 32.99 & 11.80 & 11.58 & 11.69 \\
\hline S. Em \pm & 2.11 & 1.95 & 1.84 & 2.97 & 2.34 & 2.39 & 0.59 & 0.48 & 0.34 \\
\hline C.D. at 5\% & NS & NS & NS & NS & NS & NS & NS & NS & NS \\
\hline \multicolumn{10}{|l|}{ Weed management practices } \\
\hline Weed free & 31.99 & 30.94 & 31.47 & 37.87 & 35.86 & 36.87 & 13.25 & 11.80 & 12.53 \\
\hline Hand weeding @ 20 DAS $f b$ IC at 35 DAT & 31.58 & 29.10 & 30.34 & 35.85 & 34.47 & 35.16 & 12.01 & 11.43 & 11.72 \\
\hline Pendimethalin 38.7 CS @ $0.34 \mathrm{~kg} a . i . \mathrm{ha}^{-1}$ as PE $f b$ IC at 35 DAT & 23.07 & 20.98 & 22.02 & 28.48 & 28.48 & 28.48 & 11.53 & 11.18 & 11.36 \\
\hline Oxadiargyl @ $0.04 \mathrm{~kg}_{a} a . i \mathrm{ha}^{-1}$ as PE $f b$ IC at 35 DAT & 16.86 & 15.85 & 16.35 & 27.93 & 27.83 & 27.88 & 11.08 & 10.81 & 10.94 \\
\hline S. Em \pm & 2.09 & 1.66 & 1.64 & 3.43 & 2.73 & 2.88 & 0.67 & 0.68 & 0.50 \\
\hline C.D. at 5\% & 6.02 & 4.77 & 4.73 & NS & NS & NS & NS & NS & NS \\
\hline
\end{tabular}


Table.3 Actinomycetes population (No. $\times 10^{4} \mathrm{CFU} \mathrm{g}{ }^{-1}$ of soil) at different growth stages as influenced by weed management practices in transplanted $B t$ cotton with minor millet intercropping system

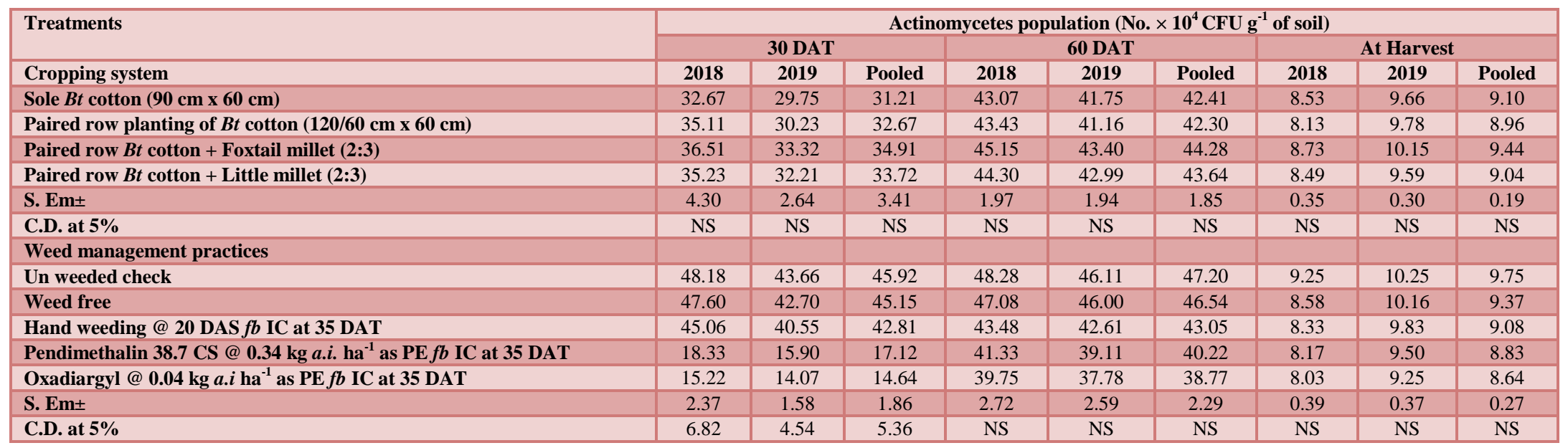

Table.4 Soil Dehydrogenase ( $\mu \mathrm{g}$ TPF formed $\mathrm{g}^{-1}$ soil day $^{-1}$ ) activity at different growth stages as influenced by weed management practices in transplanted $B t$ cotton with minor millet intercropping system

\begin{tabular}{|c|c|c|c|c|c|c|c|c|c|}
\hline \multirow{3}{*}{$\begin{array}{l}\text { Treatments } \\
\text { Cropping system }\end{array}$} & \multicolumn{8}{|c|}{ Soil Dehydrogenase ( $\mu \mathrm{g}$ TPF formed $\mathrm{g}^{-1}$ soil day ${ }^{-1}$ ) activity } & \multirow[b]{3}{*}{ Pooled } \\
\hline & \multicolumn{3}{|c|}{30 DAT } & \multicolumn{3}{|c|}{$60 \mathrm{DAT}$} & \multicolumn{2}{|c|}{ At Harvest } & \\
\hline & 2018 & 2019 & Pooled & 2018 & 2019 & Pooled & 2018 & 2019 & \\
\hline Sole $B t$ cotton $(90 \mathrm{~cm} \times 60 \mathrm{~cm})$ & 28.41 & 27.58 & 28.00 & 33.59 & 32.57 & 33.08 & 28.77 & 27.38 & 28.07 \\
\hline Paired row planting of $B t$ cotton $(120 / 60 \mathrm{~cm} \times 60 \mathrm{~cm})$ & 28.08 & 27.23 & 27.66 & 33.34 & 32.54 & 32.94 & 28.90 & 27.32 & 28.11 \\
\hline Paired row $B t$ cotton + Foxtail millet $(2: 3)$ & 29.03 & 28.18 & 28.60 & 33.99 & 33.02 & 33.51 & 29.29 & 28.05 & 28.67 \\
\hline Paired row $B t$ cotton + Little millet (2:3) & 28.54 & 27.63 & 28.09 & 33.62 & 32.63 & 33.13 & 29.11 & 27.44 & 28.27 \\
\hline S. Em \pm & 0.74 & 0.65 & 0.69 & 0.70 & 0.60 & 0.65 & 0.60 & 0.47 & 0.52 \\
\hline C.D. at $5 \%$ & NS & NS & NS & NS & NS & NS & NS & NS & NS \\
\hline \multicolumn{10}{|l|}{ Weed management practices } \\
\hline Un weeded check & 30.33 & 29.31 & 29.82 & 34.78 & 33.61 & 34.19 & 29.93 & 28.27 & 29.10 \\
\hline Weed free & 29.46 & 28.91 & 29.19 & 34.26 & 33.45 & 33.85 & 29.43 & 27.94 & 28.68 \\
\hline Hand weeding @ 20 DAS $f b$ IC at 35 DAT & 28.54 & 27.56 & 28.05 & 33.22 & 32.47 & 32.84 & 28.53 & 27.33 & 27.93 \\
\hline Pendimethalin 38.7 CS @ $0.34 \mathrm{~kg} a . i . \mathrm{ha}^{-1}$ as PE $f b$ IC at 35 DAT & 27.15 & 26.35 & 26.75 & 33.14 & 32.03 & 32.59 & 28.59 & 27.22 & 27.90 \\
\hline 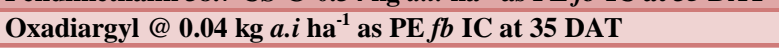 & 27.10 & 26.14 & 26.62 & 32.78 & 31.89 & 32.34 & 28.62 & 26.98 & 27.80 \\
\hline S. Em \pm & 0.66 & 0.79 & 0.72 & 0.75 & 0.78 & 0.76 & 0.72 & 0.72 & 0.71 \\
\hline C.D. at 5\% & 1.91 & 2.28 & 2.06 & NS & NS & NS & NS & NS & NS \\
\hline
\end{tabular}


Table.5 Cotton equivalent yield influenced by weed management practices in transplanted $B t$ cotton with minor millet intercropping system

\begin{tabular}{|c|c|c|c|}
\hline \multirow[t]{2}{*}{ Treatments } & \multicolumn{3}{|c|}{ Cotton equivalent yield $\left(\mathrm{kg} \mathrm{ha}^{-1}\right)$} \\
\hline & 2018 & 2019 & Pooled \\
\hline \multicolumn{4}{|l|}{ Main plot } \\
\hline Sole $B t$ cotton $(90 \mathrm{~cm} \times 60 \mathrm{~cm})\left(C_{1}\right)$ & 1771 & 1699 & 1735 \\
\hline Paired row planting of $B t$ cotton $(120 / 60 \mathrm{~cm} \times 60 \mathrm{~cm})\left(\mathrm{C}_{2}\right)$ & 1730 & 1667 & 1699 \\
\hline Paired row $B t$ cotton + Foxtail millet $(2: 3)\left(C_{3}\right)$ & 2508 & 2547 & 2528 \\
\hline Paired row $B t$ cotton + Little millet $(2: 3)\left(C_{4}\right)$ & 2182 & 2200 & 2191 \\
\hline S. $\mathbf{E m} \pm$ & 18 & 11 & 12 \\
\hline C.D. at $5 \%$ & 53 & 33 & 36 \\
\hline \multicolumn{4}{|l|}{ Sub plot } \\
\hline Un weeded check $\left(W_{1}\right)$ & 1073 & 980 & 1027 \\
\hline Weed free $\left(W_{2}\right)$ & 2528 & 2552 & 2540 \\
\hline Hand weeding @ 20 DAS $f b$ IC at 35 DAT $\left(W_{3}\right)$ & 2342 & 2365 & 2353 \\
\hline $\begin{array}{l}\text { Pendimethalin } 38.7 \text { CS @ } 0.34 \mathrm{~kg} \text { a.i. ha }{ }^{-1} \text { as PE } f b \text { IC at } 35 \\
\text { DAT }\left(W_{4}\right)\end{array}$ & 2307 & 2352 & 2330 \\
\hline Oxadiargyl @ $0.04 \mathrm{~kg} a . i \mathrm{ha}^{-1}$ as PE $f b$ IC at 35 DAT $\left(W_{5}\right)$ & 1988 & 1894 & 1941 \\
\hline S. $\mathbf{E m} \pm$ & 16 & 19 & 13 \\
\hline C.D. at $5 \%$ & 47 & 56 & 38 \\
\hline \multicolumn{4}{|l|}{ Interaction } \\
\hline $\mathbf{C}_{1} \mathbf{W}_{1}$ & 892 & 748 & 820 \\
\hline $\mathbf{C}_{1} \mathbf{W}_{2}$ & 2140 & 2120 & 2130 \\
\hline $\mathbf{C}_{1} \mathbf{W}_{3}$ & 2047 & 2037 & 2042 \\
\hline $\mathbf{C}_{1} \mathbf{W}_{4}$ & 2034 & 2032 & 2033 \\
\hline $\mathbf{C}_{1} \mathbf{W}_{5}$ & 1744 & 1559 & 1651 \\
\hline $\mathbf{C}_{2} \mathbf{W}_{1}$ & 782 & 709 & 745 \\
\hline $\mathbf{C}_{2} \mathbf{W}_{2}$ & 2091 & 2087 & 2089 \\
\hline $\mathrm{C}_{2} \mathbf{W}_{3}$ & 2037 & 2032 & 2034 \\
\hline $\mathbf{C}_{2} \mathbf{W}_{4}$ & 2034 & 2027 & 2031 \\
\hline $\mathrm{C}_{2} \mathrm{~W}_{5}$ & 1708 & 1480 & 1594 \\
\hline $\mathrm{C}_{3} \mathbf{W}_{1}$ & 1323 & 1279 & 1301 \\
\hline $\mathbf{C}_{3} \mathbf{W}_{2}$ & 3009 & 3078 & 3044 \\
\hline $\mathbf{C}_{3} \mathbf{W}_{3}$ & 2887 & 2995 & 2941 \\
\hline $\mathrm{C}_{3} \mathrm{~W}_{4}$ & 2873 & 2991 & 2932 \\
\hline $\mathrm{C}_{3} \mathrm{~W}_{5}$ & 2445 & 2394 & 2419 \\
\hline $\mathrm{C}_{4} \mathrm{~W}_{1}$ & 1296 & 1184 & 1240 \\
\hline $\mathbf{C}_{4} \mathbf{W}_{2}$ & 2873 & 2923 & 2898 \\
\hline $\mathrm{C}_{4} \mathbf{W}_{3}$ & 2398 & 2394 & 2396 \\
\hline $\mathrm{C}_{4} \mathbf{W}_{4}$ & 2288 & 2358 & 2323 \\
\hline $\mathrm{C}_{4} \mathrm{~W}_{5}$ & 2055 & 2144 & 2099 \\
\hline S. $\mathbf{E m} \pm$ & 27 & 28 & 21 \\
\hline C.D. at $5 \%$ & 77 & 80 & 60 \\
\hline
\end{tabular}

Plate.1 Bacteria, Fungi and Actinomycetes population of soil collected at 30 days after treatment incorporation

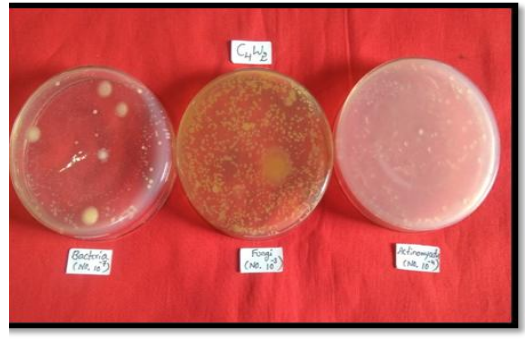

Soil collected from weed free treatment

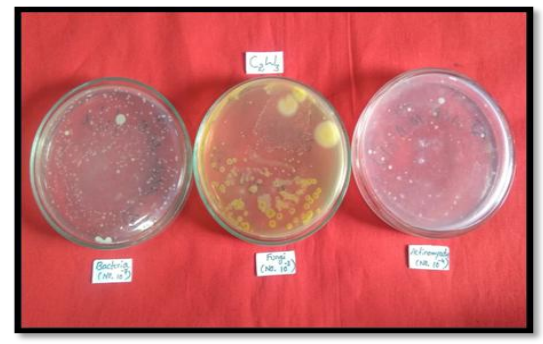

Soil collected from hand weeding treatment 


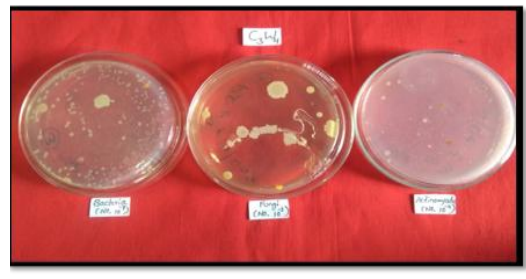

Soil collected from pendimethalin sprayed treatment

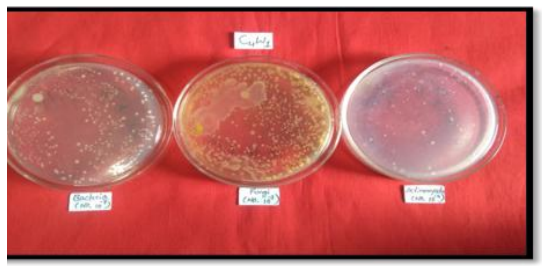

il collected from un weeded check treatment

The trend remained same during both the years showing no pendimethalin and oxadiargyl herbicide residue present in the soil at toxic level indicating degradation of the herbicide in the soil. The results were confirmed with the findings of Kaur et al., (2014) and Trimurthulu et al., (2015).

\section{Dehydrogenase enzyme activity ( $\mu$ g TPF formed $\mathrm{g}^{-1}$ soil day $^{-1}$ )}

Dehydrogenase enzyme activity in soil is used as an indicator of soil biological (microbial) activity in soil because it is an intracellular enzyme in all living microbial cells (Quilchano and Maranon, 2002).

Dehydrogenase activity of soil did not differ significantly due to cropping systems at different growth stages of $B t$ cotton during both the years of experimentation.

Among weed management practices, the pooled data at 30 DAT indicated that application of pendimethalin 38.7 CS @ $0.34 \mathrm{~kg}$ a.i. ha ${ }^{-1}$ or oxadiargyl @ $0.04 \mathrm{~kg}$ a.i. $\mathrm{ha}^{-1}$ as pre emergence recorded Dehydrogenase activity of $26.75 \mu \mathrm{g}$ TPF formed $\mathrm{g}^{-1}$ soil day ${ }^{-1}$ and $26.62 \mu \mathrm{g}$ TPF formed

$\mathrm{g}^{-1}$ soil day $^{-1}$, respectively. These values

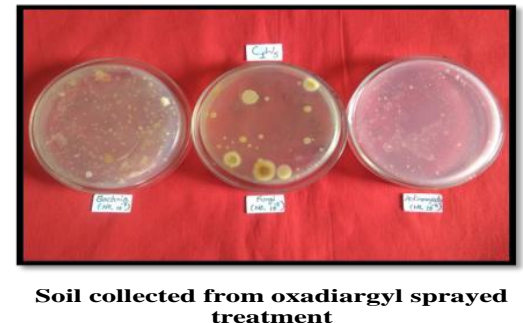

treatment were significantly lower over Dehydrogenase activity recorded in unweeded check (29.82 $\mu \mathrm{g}$ TPF formed $\mathrm{g}^{-1}$ soil day $\left.{ }^{-1}\right)$, weed free check (29.19 $\mu \mathrm{g}$ TPF formed $\mathrm{g}^{-1}$ soil day $^{-1}$ ) and hand weeding @ 20 DAT $f b$ IC at 35 DAT $(28.05 \mu \mathrm{g}$ TPF formed $\mathrm{g}^{-1}$ soil day $^{-1}$ ). The latter treatments were on par with each other. The trend remained same during both the years. The data was non significant at 60 DAT and at harvest. This clearly indicated that though application of pendimethalin or oxadiargyl reduced the Dehydrogenase activity immediately after application but its effect did not persists longer in soil (Table 4).

\section{Cotton equivalent yield}

Cotton equivalent yield (CEY) varied significantly due to cropping systems with weed management practices. Among the cropping systems, in pooled data significantly higher CEY was recorded in paired row $B t$ cotton + foxtail millet $(2: 3)$ $\left(2528 \mathrm{~kg} \mathrm{ha}^{-1}\right.$ ) followed by paired row $B t$ cotton + little millet $(2: 3)$ (2191 kg ha-1). This was followed by sole $B t$ cotton (1735 $\mathrm{kg} \mathrm{ha}^{-1}$ ) which inturn on par with paired row sole $B t$ cotton $\left(1699 \mathrm{~kg} \mathrm{ha}^{-1}\right)$. The results suggest that though the intercrops reduced the yield of cotton, the total productivity 
would be high in intercropping system. There will be better utilization of other resources like light, nutrients and moisture. These results confirm the findings of Aladakatti et al., (2011).

In pooled data, among weed management practices, weed free check recorded significantly higher seed cotton equivalent yield $\left(2540 \mathrm{~kg} \mathrm{ha}^{-1}\right)$ over rest of the treatments. This was followed by hand weeding @ 20 DAT $f b$ IC at 35 DAT (2353 $\mathrm{kg} \mathrm{ha}^{-1}$ ) and pendimethalin 38.7 CS @ 0.34 $\mathrm{kg}$ a.i. ha ${ }^{-1}$ as PE $f b$ IC at 35 DAT $(2330 \mathrm{~kg}$ $\mathrm{ha}^{-1}$ ) which were on par with each other but significantly higher over oxadiargyl @ 0.04 $\mathrm{kg}$ a.i. ha ${ }^{-1}$ as PE $f b$ IC at 35 DAT $(1941 \mathrm{~kg}$ $\mathrm{ha}^{-1}$ ).

The significantly lower seed cotton equivalent yield was recorded in unweeded check (1027 kg ha $\left.{ }^{-1}\right)$. The trend remains same during both the years. The increased seed cotton equivalent yield hectare ${ }^{-1}$ might be due to better control of weeds during critical period of crop weed competition over unweeded check treatment that inturn improved the growth and yield parameters of $B t$ cotton and foxtail millet. The results were confirmed with the findings of Giri et al., (2006). There was no suppression of yield due to herbicide application indicating no residual effect.

Further, in pooled data interaction between cropping systems and weed management practices, paired row $B t$ cotton + foxtail millet (2:3) coupled with weed free treatment $\left(\mathrm{C}_{3} \mathrm{~W}_{2}\right)$ recorded significantly higher CEY (3044 kg ha ${ }^{-1}$ ) closely followed by paired row $B t$ cotton + foxtail millet $(2: 3)$ in combination with hand weeding @20 DAT $f b$ IC at 35 DAT (2941 kg ha-1), pendimethalin 38.7 CS @ $0.34 \mathrm{~kg}$ a.i. ha ${ }^{-1}$ as PE $f b$ IC at 35 DAT (2932 kg ha ${ }^{-1}$ ) and paired row $B t$ cotton + little millet with weed free treatment $\left(2898 \mathrm{~kg} \mathrm{ha}^{-1}\right.$ ) (Table $5)$. These treatments were on par with each other but significantly higher CEY over rest of the combinations. This clearly indicate that these herbicides can be used in these intercropping systems without any adverse effect of herbicide.

In conclusion the pre emergence application of pendimethalin $38.7 \mathrm{CS} @ 0.34 \mathrm{~kg}$ a.i. ha ${ }^{-1}$ and oxadiargyl @ $0.04 \mathrm{~kg}$ a.i. ha ${ }^{-1}$ had temporarily reduction in number of soil Bacteria, Fungi, Actinomycetes and Dehydrogenase activity but later it was recovered showing no residual effect. The seed cotton equivalent yield was significantly higher in cultural and herbicide treated plots indicating these herbicides can be used for control of weeds.

\section{References}

Aladakatti, Y. R., Hallikeri, S. S., Nandagavi, R. A., Hugar, A. Y. and Naveen, N. E., 2011, Effect of intercropping of oilseed crops on growth, yield and economics of cotton (Gossypium hirsutum) under rainfed conditions. Karnataka J. Agric. Sci., 24(3): 280-282.

Casida, L., Klein, D. and Santoro, T., 1964, Soil Dehydrogenase activity. Soil Sci., 98: 371-376.

Giri, A. N., Deshmukh, M. N. and Gore, S. B., 2006, Effect of cultural and integrated methods of weed control on cotton, intercrop yield and weedcontrol efficiency in cotton based cropping systems. Indian $J$. Agron., 51(1): 34-36.

Gomez, K. A. and Gomez, A. A., 1984, Statistical Procedures for Agricultural Research, $2^{\text {nd }}$ Editn. A wiley Inter-Science Publications, New York (USA).

Kaur, S., Singh, S. and Phutela, R. P., 2014, 
Effect of herbicides on soil microorganisms. Indian J. Weed Sci., 46: 229-223.

Pramer, D. and Schmidt, E. L., 1964, Experimental soil microbiology. Burgess Pub. Co., Minneapolis, US.

Quilchano, C. and Maranon, T., 2002, Dehydrogenase activity in Mediterranean forest Soils. Biol.
Fert. Soils, 35: 102-107. Trimurthulu, N., Ashok, S., Latha, M. and Rao, A. S., 2015, Influence of preemergence herbicides on the soil microflora during the crop growth of black gram (Vigna mungo L). Int. J. Curr. Microbiol. Appl. Sci., 4: 539546.

\section{How to cite this article:}

Siddagangamma, K. R., A. S. Channabasavanna, Mahadevaswamy, K. Narayana Rao, M. Y. Ajayakumar and Yadahalli, G. S. 2021. Effect of Herbicides on Soil Microflora and Dehydrogenase Activity in Transplanted Bt Cotton Based Intercropping System. Int.J.Curr.Microbiol.App.Sci. 10(01): 902-909. doi: https://doi.org/10.20546/ijcmas.2021.1001.108 